\title{
Surgical Treatment of Chronic Periprosthetic Joint Infection: Fate of Spacer Exchanges
}

Running Title: Fate of Spacer Exchanges in PJI

\author{
Timothy L. Tan MD ${ }^{1}$ \\ Karan Goswami MD ${ }^{1}$ \\ Michael M. Kheir MD ${ }^{1,2}$ \\ Chi Xu MD ${ }^{1}$ \\ Qiaojie Wang MD ${ }^{1,3}$ \\ Javad Parvizi MD FRCS ${ }^{1}$
}

${ }^{1}$ Rothman Orthopaedic Institute at Thomas Jefferson University, Philadelphia, PA

${ }^{2}$ Indiana University School of Medicine, Indianapolis, IN

${ }^{3}$ Shanghai Jiao Tong University, Shanghai, China

\section{Corresponding Author}

Javad Parvizi MD, FRCS

Rothman Orthopaedic Institute

$125 \mathrm{~S} 9^{\text {th }}$ St. Ste 1000

Philadelphia, PA 19107

P: 267-339-7813

F: 215-503-5651

parvj@aol.com

This is the author's manuscript of the article published in final edited form as:

Tan, T. L., Goswami, K., Kheir, M. M., Xu, C., Wang, Q., \& Parvizi, J. (2019). Surgical Treatment of Chronic Periprosthetic Joint Infection: Fate of Spacer Exchanges. The Journal of Arthroplasty. https://doi.org/10.1016/j.arth.2019.04.016 


\section{ABSTRACT}

Introduction: Patients with periprosthetic joint infection (PJI) undergoing two-stage exchange arthroplasty may undergo an interim spacer exchange for a variety of reasons including mechanical failure of spacer or persistence of infection. The objective of this study was to understand the risk factors and outcomes of patients that undergo spacer exchange during the course of a planned two-stage exchange arthroplasty.

Methods: Our institutional database was used to identify 533 patients who underwent a two-stage exchange arthroplasty for PJI, including 90 patients with a spacer exchange, from 2000-2017. A retrospective review was performed to extract relevant clinical information. Treatment outcomes included 1) progression to reimplantation and 2) treatment success as defined by a Delphi-based criterion. Both univariate and multivariate COX regression models were performed to investigate whether spacer exchange was associated with failure. Additionally, a propensity score analysis was performed based on a 1:2 match.

Results: A spacer exchange was required in $16.9 \%$. Patients who underwent spacer exchanges had a higher body mass index (BMI) $(\mathrm{p}<0.001)$, rheumatoid arthritis $(\mathrm{p}=0.018)$, and were more likely to have PJI caused by resistant $(0.048)$ and polymicrobial organisms $(\mathrm{p}=0.007)$. Patients undergoing a spacer exchange demonstrated lower survivorship and an increased risk of failure in the multivariate and propensity score matched analysis compared to patients who did not require a spacer exchange.

Discussion: Despite an additional load of local antibiotics and repeat debridement, patients who underwent a spacer exchange demonstrated poor outcomes, including failure to undergo reimplantation and twice the failure rate. The findings of this study may need to be borne in mind when managing patients who require spacer exchange. 
27 Keywords: Periprosthetic Joint infection, knee, hip, infection, spacer exchange, two-stage 28 exchange

29 


\section{INTRODUCTION}

Treatment of periprosthetic joint infection (PJI) after total joint arthroplasty (PJI) remains a challenge with a high failure rate[1,2]. Two-stage exchange arthroplasty is the most frequent treatment for chronic PJI, involving removal of the components and insertion of an antibiotic impregnated cement spacer in the first stage and reimplantation of permanent implants at a later stage $[3,4]$. Outcomes after two stage exchange arthroplasty remain far from perfect as many patients are not ultimately reimplanted and multiple surgeries are frequently required to eradicate infection[2,5,6].

There are occasions when the initial antibiotic cement spacer may be exchanged, which is termed by some as the "three stage exchange" as it involves an additional surgical procedure. Reasons for a spacer exchange may include persistent infection or a fractured or dislocated spacer $[2,7,8]$. In patients with persistent infection, the rationale behind a spacer exchange is to deliver an additional load of local antibiotics and to repeat surgical debridement to treat the persistent infection.[9-11] Although this practice has been adopted by some surgeons, there is minimal literature on the outcomes of spacer exchange. Understanding the outcomes of spacer exchanges is important as a spacer exchange further delays reimplantation and subjects the patient to an additional surgery and all the morbidities associated with it.

The aim of this study was to report the prevalence, characteristics and outcomes of patients with PJI who required a spacer exchange during the course of their intended two-stage exchange arthroplasty. We also intended to compare the outcome of these patients with those undergoing conventional two-stage revision without an interim spacer exchange. 


\section{MATERIALS AND METHODS}

A retrospective institutional study was performed to identify all patients with PJI who underwent a two-stage exchange arthroplasty from January 2000 to May 2017. The diagnosis of PJI was based on the Musculoskeletal Infection Society (MSIS) and the International Consensus Meeting criteria[12,13]. Patients with a megaprosthesis, initial infection with a fungal organism, prior native septic arthritis, or prior failed two-stage exchange arthroplasty were excluded. We also excluded 80 patients with reimplantation due to follow-up less than 1 year after reimplantation and 18 patients without eventual reimplantation by May 2018 due to lost to follow-up after the last spacer insertion. After the aforementioned criteria, 533 joints (203 hips and 330 knees) were included in the final analysis. Of these 533 joints, 90 patients ( 31 hips and 59 knees) underwent an initial interim spacer exchange during the course of their two-stage revision treatment (exchange group). This cohort was compared with a control group of 443 PJIs (172 hips and 271 knees) that did not undergo an interim spacer exchange (Figure 1).

A retrospective review was performed to extract relevant information regarding surgical treatment, microbiology during resection arthroplasty, demographic data (age, body mass index [BMI], gender), Charlson comorbidity index (CCI) [15], diabetes, rheumatoid arthritis, index surgery (primary or revision), prior irrigation and debridement (I\&D) on the same joint, the presence of a sinus tract, follow-up time, date of surgery, and antibiotics used in the spacer. Both static (66.8\%) and articulating spacers (33.2\%) were utilized containing dual antibiotics against both gram positive and gram-negative organisms; 1 to $3 \mathrm{~g}$ of vancomycin and 1 to $3.6 \mathrm{~g}$ of tobramycin per 40-gram pack of bone cement was used almost exclusively (98.3\%). The articulating spacers were intraoperatively constructed primarily from prefabricated molds with endoskeleton implants. The decision to undergo reimplantation was based on trending of serum 
inflammatory markers and a healing wound. Routine aspiration prior to reimplantation was not performed. In patients in whom there was suspicion of continued infection, such as poor wound healing, intraoperative purulence, or mechanical spacer issues, it was institutional protocol for a repeat spacer to be performed in order to introduce a new load of antibiotics. The decision to perform multiple spacers exchanges rather than undergo salvage surgery with a girdlestone, or fusion was based on a shared decision between the patient and surgeon. Following reimplantation, patients were routinely suppressed with antibiotics starting in 2016.

The primary endpoints of this study were 1) failure to ultimately undergo reimplantation, and 2) treatment failure after reimplantation as assessed by the Delphi method-based criteria by DiazLedezma [7]. The latter endpoint was defined as: 1) failed infection eradication, characterized by the presence of a sinus tract, drainage, pain, or infection recurrence caused by the same organism strain; 2) subsequent surgical intervention for infection after reimplantation surgery; or 3) occurrence of PJI-related mortality[16]. Patients on suppression were not considered a failure.

Failure was only evaluated after reimplantation to ensure that the starting point was the same for both groups and to comply with the aforementioned definition of success.

\section{Statistical Analysis}

All of the statistical analyses were performed with the statistical software package $\mathrm{R}$ (http://www.R-project.org, The R Foundation). The clinical characteristics between groups were compared with the use of the independent t-test or Mann-Whitney test for continuous variables and the chi-square test or Fisher's exact test for categorical variables. Kaplan-Meier survivorship curves were generated to compare outcomes and a log-rank test was performed to assess statistical significance. Both univariate and multivariate logistic regression models were performed to investigate whether spacer exchange was associated with failure to reimplantation 
and Cox regression models were conducted to identify the relationship between spacer exchange and treatment failure. In the multivariate model, we adjusted all variables included in Table $\mathbf{1}$. Results were presented as odds ratios (OR) or hazards ratio (HR) with $95 \%$ confidence intervals $(\mathrm{CI})$.

\section{Sensitivity Analysis}

A set of sensitivity analysis was performed using propensity score matching (PSM), which can adjust for some baseline group differences and is a well-accepted method to account for identified confounding variables $[17,18]$. Propensity scores of spacer exchange (vs. no spacer exchange) were estimated by logistic regression using age, gender, BMI, joints, CCI score, index surgery, diabetes, rheumatoid arthritis, the present of a sinus tract, prior I\&D on the same joint, resistant organisms (Methicillin Resistant Staphylococcus Aureus (MRSA) or Vancomycin Resistant Enterococcus (VRE)), polymicrobial organisms, and duration of follow-up. Patients who underwent a spacer exchange were matched 1:2 (without a spacer exchange) on the logit of the propensity score using a nearest-neighbor matching approach. The maximum difference between propensity probabilities for matching was set at 0.2 . A standardized mean difference (SMD) for each covariate was used to examine the balance of covariates between patients who received a spacer exchange and matched control individuals. PSM score was adjusted in the multivariate model. For all statistical analyses, significance was set at an alpha of 0.05 .

\section{RESULTS}

Patient demographics and culture results at the initial spacer implantation are shown in

Table 1. One or more spacer exchanges were required in $16.9 \%$ of two stage exchange arthroplasties (90/533). Patients in the spacer exchange group had a higher mean body mass 
index (BMI) $\left(34.4 \pm 8.5\right.$ vs. $\left.31.4 \pm 8.0 \mathrm{~kg} / \mathrm{m}^{\wedge} 2, \mathrm{p}<0.001\right)$ and percentage of rheumatoid arthritis

121 (14.6\% vs. $7.0 \%, \mathrm{p}=0.018)$ compared to the control group. S. aureus was the predominant

122

123

124

125

126

organism in both the spacer exchange and the control group ( $36.7 \%$ vs. $39.3 \%, p=0.643)$. The prevalence of PJI caused by resistant organisms ( $23.3 \%$ vs. $14.9 \%, \mathrm{p}=0.048)$ and polymicrobial organisms $(18.9 \%$ vs. 9.3\%, $\mathrm{p}=0.007)$ were significantly higher in the spacer exchange group compared to controls. Of the patients with persistent infection, the organism was same between the spacer exchange and initial spacer insertion in $11.5 \%$ of patients, all of which were antibiotic resistant organisms (MRSA or VRE).

Seventy-nine patients had only 1 spacer exchange ( 2 spacers total), 8 patients had 2 spacer exchanges ( 3 spacers total), 2 patients had 3 spacer exchanges ( 4 spacers total), and 1 patient had 4 spacer exchange ( 5 spacers total). The reasons for the initial spacer exchange included suspected persistence of infection (74/90), spacer dislocation (7/90), and fracture or unknown reasons $(9 / 90)$.

Of the 533 intended two stage exchange arthroplasties, the overall reimplantation rate was $79.7 \%(425 / 533)$. The reimplantation rate was $70.0 \%(63 / 90)$ for patients with at least one spacer exchange compared to $81.7 \%$ (362/443) for those without spacer exchange. After adjusting all confounders, patients with a spacer exchange were at an increased risk of failure to undergo reimplantation (OR, 1.96; 95\% CI, 1.08 to 3.53; Table 2). The reasons for not undergoing reimplantation among 27 patients in the spacer exchange group were: medically unfit for reimplantation ( $\mathrm{n}=11$ ), salvage procedures for persistent infection (5 fusion, 3 amputation and 1 girdlestone), death during stages $(n=3)$, and decision to retain spacer either by the patient or the surgeon $(n=4)$. 
Following reimplantation, the overall treatment success rate according to the Delphia-

143

144

145

146

147

148

149

150

151

152

153

154

155

156

157

158

159

160

161

162

163

164 based definition was 75.1\% (319/425) with a mean follow-up of 5.1 year (range 1.0 to 16.2 years). The reinfection rate was $41.3 \%$ (26/63) for patients with spacer exchange compared to $22.1 \%(80 / 362)$ for those without spacer exchange. In patients with a spacer exchange for mechanical failure, the failure rate after reimplantation was $33.33 \%(4 / 12)$ compared to $43.14 \%$ $(22 / 51)$ in patients who underwent an exchange for infection $(\mathrm{p}=0.746)$ and $22.10 \%(80 / 362)$ in those without a reoperation $(\mathrm{p}=0.479)$. After adjusting all confounders, the reinfection rate in patients with spacer exchange was significantly higher than controls (HR, 2.05; 95\% CI, 1.08 to 3.89; Table 3). Kaplan-Meier survivorship curves also revealed a significantly lower treatment success in the spacer-exchange group compared to controls using log-rank test $(p<0.001$, Figure 2). The results were similar when isolating only patients that received a spacer exchange for infection; Kaplan-Meier survivorship curve revealed significantly lower treatment success rates in this stratified cohort as compared to controls ( $p<0.001$, Figure 3). When stratified by joint, survivorship was lower in patients with a spacer exchange compared to those without a spacer exchange with treatment failure as an endpoint for both THAs (Figure 4) and TKAs (Figure 5). Through using propensity score matching (PSM), we generated a subsample of 88 cases with a spacer exchange and 176 matched controls without a spacer exchange. The patient characteristics after matching were shown in Appendix Table 1 and the quality of PSM was considered balanced (all SMD<0.2). Patients with a spacer exchange did not demonstrate a higher rate of failure to undergo reimplantation in the propensity score analysis (PSM scoreadjusted OR, 1.44 ; $95 \%$ CI, 0.80 to 2.60 ; Table 2). The relationship between spacer exchange and subsequent reinfection remained robust; reinfection rate in patients with spacer exchange was significantly higher than matched controls (PSM score-adjusted HR, 2.23; 95\% CI, 1.14 to 
4.40; Table 3). Kaplan-Meier survivorship curves revealed a significantly lower treatment success in the spacer-exchange group compared to matched controls ( $\mathrm{p}=0.007$, Appendix Figure 1). When isolating only patients that received a spacer exchange for infection, the results did not change ( $\mathrm{p}=0.006$, Appendix Figure 2).

\section{DISCUSSION}

A spacer exchange for persistent infection or spacer-related mechanical complications such as fracture or dislocation may be performed in patients undergoing two-stage exchange arthroplasty. In the current study, $16.9 \%$ of patients who underwent an intended two-stage exchange arthroplasty had an interim spacer exchange. The primary reason of spacer exchange was suspicion of persistent infection. These patients were more likely to have obesity, rheumatoid arthritis, or PJI caused by resistant and/or polymicrobial organisms compared to those without a spacer exchange. Interestingly, spacer exchange was associated with an increased risk of reinfection following reimplantation regardless of whether the exchange was done for mechanical failure of the spacer or suspicion for persistence of infection. These findings continued to be present after the propensity score analysis which matched for baseline differences in comorbidities.

To our knowledge, only one other study has specifically investigated outcomes after spacer exchanges[10]. In a series of 347 two stage exchanges, including 59 spacer exchanges, George et al. found that patients who underwent spacer exchanges had decreased survivorship $(\mathrm{p}=0.020)$ after reimplantation[10]. In addition, the spacer exchange group demonstrated increased comorbidities, and an increased prevalence of resistance organisms. Our results are 
consistent with the prior study in demonstrating a poor outcome for patients undergoing an interim spacer exchange.

There are several possibilities that may explain the poor outcome in patients with a spacer exchange. The most likely reason is that the patients may be poor hosts with increased comorbidities and/or difficult to eradicate organisms (e.g. resistant or polymicrobial) which may predispose the patient to persistent infection[19-21]. However, even in the multivariate and propensity score analysis, patients who underwent a spacer exchange, including those for mechanical failure of spacer, were more likely to have subsequent treatment failure. Thus, it is possible that the increased risk of treatment failure in patients undergoing spacer exchange may be the result of catabolic burden and morbidity that an additional surgery carries. This may be particularly true in patients with extensive comorbidities. In fact recognizing the issues related to an additional surgery, the Second International Consensus Meeting on Orthopedic Infections (ICM) recommends that patients with mechanical failure of a spacer should not undergo an additional spacer exchange unless the failed spacer results in soft tissue problems[11]. Regardless of the reason for the increased risk of failure and poor outcome, the present study suggests that the frequent treatment of a persistent infection after a two-stage exchange with an additional repeat spacer demonstrates poor outcomes and that the utility of this treatment method should be reconsidered.

Another important issue to examine is that patients who failed after a two-stage exchange arthroplasty or were suspected of having a persistent infection are more likely to be infected with more virulent organisms such as Staphylococcal species and/or resistant organisms [5,22,23,24], We found similar results in this study, with Staphylococcal species comprising the majority of persistent infections during the first spacer exchange followed closely by other resistant 
organisms. While subsequent surgery after failure of a two-stage exchange demonstrate poor

211 outcomes in the literature, we found that patients undergoing spacer exchanges mirror these results with a high rate of salvage procedures.

There are several limitations to this study that should be considered. First, the study is retrospective in nature and thus relies on accurate and detailed documentation. This limitation is particularly important when evaluating the reason for not undergoing reimplantation, as this was infrequently recorded in the medical record. In addition, although clinical signs and improvement are also used as a proxy for infection control, this information is difficult to obtain in a retrospective study. Furthermore, there were differences in baseline characteristics which may result from a selection bias as it is feasible that a surgeon is more aggressive and more likely to perform a spacer exchange in patients with increased comorbidities and/or PJI caused by resistant organisms. However, we attempted to control for these baseline differences using both a multivariate analysis and propensity score matching based analysis. In addition, the influence of antibiotic suppression could not be controlled for as this information was not readily available. Furthermore, while we found that patients that underwent a spacer exchange were more likely to have rheumatoid arthritis, we were unable to investigate the influence of anti-rheumatic medication, including the role of modern disease-modifying antirheumatic drugs, which selectively target the immune system. Additionally, many patients were lost to follow up as many of these patients were referred from an outside hospital and follow-up with their original surgeon whose records are not readily available. This may thus reflect an underestimation of the true failure rate. Lastly, it was routine protocol to perform a spacer exchange rather than a 231 girdlestone at our institution with the intent of introducing more local antibiotics. We 232 acknowledge that there is no clear consensus regarding the optimal management of persistent 
233 infection in the setting of a spacer and that some surgeons will resort to an "implant holiday"

234 prior to an intended reimplantation.

235 In summary, the present study highlights the challenges that remain in managing patients

236 with persistent infection after an initial spacer implantation. Despite delivery of an additional

237 load of local antibiotics and further debridement, outcome of surgical treatment of these patients

238 remains poor and the risk of failure is actually increased. Furthermore, a significant number of

239 patients with a spacer exchange never ultimately undergo reimplantation despite being subject to

240 the morbidity of another surgery. Surgeons should be cognizant of these suboptimal outcomes

241 after treatment with an additional spacer exchange and alternative strategies are certainly needed.

242 It is crucial for subsequent studies to understand risk factors for subsequent failure of a spacer

243 exchange in order to determine the indications for a spacer exchange. 


\section{References:}

[1] Tan TL, Goswami K, Fillingham YA, Shohat N, Rondon AJ, Parvizi J. Defining Treatment Success After 2-Stage Exchange Arthroplasty for Periprosthetic Joint Infection. J Arthroplasty 2018;33:3541-6. doi:10.1016/j.arth.2018.06.015.

[2] Gomez MM, Tan TL, Manrique J, Deirmengian GK, Parvizi J. The Fate of Spacers in the Treatment of Periprosthetic Joint Infection. J Bone Joint Surg Am 2015;97:1495-502. doi:10.2106/JBJS.N.00958.

[3] Meek RMD, Masri BA, Dunlop D, Garbuz DS, Greidanus NV, McGraw R, et al. Patient Satisfaction and Functional Status After Treatment of Infection at the Site of a Total Knee Arthroplasty with Use of the PROSTALAC Articulating Spacer. J Bone Jt Surg Am 2003;85:1888-92.

[4] Scott IR, Stockley I, Getty CJ. Exchange arthroplasty for infected knee replacements. A new two-stage method. J Bone Joint Surg Br 1993;75:28-31.

[5] Fehring KA, Abdel MP, Ollivier M, Mabry TM, Hanssen AD. Repeat Two-Stage Exchange Arthroplasty for Periprosthetic Knee Infection Is Dependent on Host Grade. J Bone Jt Surg Am 2017;99:19-24. doi:10.2106/JBJS.16.00075.

[6] Kheir MM, Tan TL, Gomez MM, Chen AF, Parvizi J. Patients With Failed Prior TwoStage Exchange Have Poor Outcomes After Further Surgical Intervention. J Arthroplasty 2017;32:1262-5. doi:10.1016/j.arth.2016.10.008.

[7] Barreira P, Leite P, Neves P, Soares D, Sousa R. Preventing mechanical complications of hip spacer implantation: technical tips and pearls. Acta Orthop Belg 2015;81:344-8.

[8] Petis SM, Abdel MP, Perry KI, Mabry TM, Hanssen AD, Berry DJ. Long-Term Results of a 2-Stage Exchange Protocol for Periprosthetic Joint Infection Following Total Hip Arthroplasty in 164 Hips. J Bone Joint Surg Am 2019;101:74-84. doi:10.2106/JBJS.17.01103.

[9] Citak M, Argenson J-N, Masri B, Kendoff D, Springer B, Alt V, et al. Spacers. J Arthroplasty n.d.;29:93-9. doi:10.1016/j.arth.2013.09.042.

[10] George J, Miller EM, Curtis GL, Klika AK, Barsoum WK, Mont MA, et al. Success of Two-Stage Reimplantation in Patients Requiring an Interim Spacer Exchange. J Arthroplasty 2018;33:S228-32. doi:10.1016/j.arth.2018.03.038.

[11] Abdel M, Barreira P, Battenberg A, Berry D, Blevins K, Font-Vizcarra L, et al. Hip and Knee Section, Treatment, Two-Stage Exchange Spacer-Related: Proceedings of International Consensus on Orthopedic Infections. J Arthroplasty 2018;0. doi:10.1016/j.arth.2018.09.027.

[12] Parvizi J, Gehrke T, International Consensus Group on Periprosthetic Joint Infection. Definition of periprosthetic joint infection. J Arthroplasty 2014;29:1331. doi:10.1016/j.arth.2014.03.009.

[13] Workgroup Convened by the Musculoskeletal Infection Society. New definition for periprosthetic joint infection. J Arthroplasty 2011;26:1136-8. doi:10.1016/j.arth.2011.09.026.

[14] Zmistowski B, Della Valle C, Bauer TW, Malizos KN, Alavi A, Bedair H, et al. Diagnosis of periprosthetic joint infection. J Arthroplasty 2014;29:77-83. doi:10.1016/j.arth.2013.09.040. 
[15] Charlson ME, Pompei P, Ales KL, MacKenzie CR. A new method of classifying prognostic comorbidity in longitudinal studies: development and validation. Journal of chronic diseases. 1987;40(5):373-83. n.d.

[16] Diaz-Ledezma C, Higuera CA, Parvizi J. Success after treatment of periprosthetic joint infection: a Delphi-based international multidisciplinary consensus. Clin Orthop. 2013;471(7):2374-2382. doi:10.1007/s11999-013-2866-1. n.d.

[17] Kahlert J, Gribsholt SB, Gammelager H, Dekkers OM, Luta G. Control of confounding in the analysis phase - an overview for clinicians. Clin Epidemiol 2017;9:195-204. doi:10.2147/CLEP.S129886.

[18] Austin PC. An Introduction to Propensity Score Methods for Reducing the Effects of Confounding in Observational Studies. Multivar Behav Res 2011;46:399-424. doi:10.1080/00273171.2011.568786.

[19] Kheir MM, Tan TL, George J, Higuera CA, Maltenfort MG, Parvizi J. Development and Evaluation of a Prognostic Calculator for the Surgical Treatment of Periprosthetic Joint Infection. J Arthroplasty 2018;33:2986-2992.e1. doi:10.1016/j.arth.2018.04.034.

[20] Tan TL, Kheir MM, Tan DD, Parvizi J. Polymicrobial Periprosthetic Joint Infections: Outcome of Treatment and Identification of Risk Factors. J Bone Joint Surg Am 2016;98:2082-8. doi:10.2106/JBJS.15.01450.

[21] Marculescu CE, Cantey JR. Polymicrobial Prosthetic Joint Infections: Risk Factors and Outcome. Clin Orthop 2008;466:1397-404. doi:10.1007/s11999-008-0230-7.

[22] Zmistowski B, Tetreault MW, Alijanipour P, Chen AF, Della Valle CJ, Parvizi J. Recurrent periprosthetic joint infection: persistent or new infection? J Arthroplasty 2013;28:1486-9. doi:10.1016/j.arth.2013.02.021.

[23] Berend KR, Lombardi AV, Morris MJ, Bergeson AG, Adams JB, Sneller MA. Two-stage treatment of hip periprosthetic joint infection is associated with a high rate of infection control but high mortality. Clin Orthop 2013;471:510-8. doi:10.1007/s11999-012-2595-x.

[24] Kheir MM, Tan TL, Gomez MM, Chen AF, Parvizi J. Patients With Failed Prior TwoStage Exchange Have Poor Outcomes After Further Surgical Intervention. J Arthroplasty 2016. doi:10.1016/j.arth.2016.10.008. 
Appendix Table 1 Patient demographics after matching

\begin{tabular}{lllll}
\hline & $\begin{array}{l}\text { Exchange } \\
\text { group } \\
(\mathrm{N}=88)\end{array}$ & $\begin{array}{l}\text { Non-exchange group } \\
(\mathrm{N}=176)\end{array}$ & SMD & P-value \\
\hline Age (year) (mean $\pm \mathrm{SD})$ & $65.6 \pm 10.3$ & $66.4 \pm 10.7$ & 0.0777 & 0.555 \\
Male (\%) & $43(48.9)$ & $97(55.1 \%)$ & 0.1253 & 0.407 \\
BMI $\left(\mathrm{kg} / \mathrm{m}^{2}\right)$ & $34.4 \pm 8.5$ & $32.9 \pm 8.9$ & 0.1712 & 0.195 \\
Hip (\%) & $31(35.2)$ & $68(38.6)$ & 0.0707 & 0.686 \\
CCI score (mean $\pm \mathrm{SD})$ & $3.9 \pm 1.72$ & $4.1 \pm 1.9$ & 0.1545 & 0.247 \\
Diabetes $(\%)$ & $27(30.7)$ & $58(33)$ & 0.0488 & 0.816 \\
Rheumatoid arthritis $(\%)$ & $13(14.8)$ & $21(11.9)$ & 0.0836 & 0.649 \\
Index revision $(\%)$ & $30(34.1)$ & $56(31.8)$ & 0.0484 & 0.816 \\
Prior I\&D $(\%)$ & $24(27.3)$ & $53(30.1)$ & 0.0628 & 0.738 \\
Sinus tract $(\%)$ & $25(28.4)$ & $56(31.8)$ & 0.0744 & 0.671 \\
Resistant organism $(\%)$ & $21(23.9)$ & $40(22.7)$ & 0.0269 & 0.959 \\
Polymicrobial $(\%)$ & $16(18.2)$ & $31(17.6)$ & 0.0148 & 1.000 \\
\hline
\end{tabular}


Table 1 Patient demographics

\begin{tabular}{lccc}
\hline & $\begin{array}{l}\text { Exchange group } \\
(\mathrm{N}=90)\end{array}$ & $\begin{array}{l}\text { Non-exchange } \\
\text { group } \\
(\mathrm{N}=443)\end{array}$ & P-value \\
\hline Age (year) (mean $\pm \mathrm{SD})$ & $65.5 \pm 10.2$ & $66.0 \pm 11.4$ & 0.364 \\
Male (n, \%) & $44(49.4 \%)$ & $222(51.7 \%)$ & 0.692 \\
BMI $\left(\mathrm{kg} / \mathrm{m}^{2}\right)$ & $34.4 \pm 8.5$ & $31.4 \pm 8.0$ & $<0.001$ \\
Hip (n, \%) & $31(34.4 \%)$ & $172(38.8 \%)$ & 0.435 \\
CCI score (mean $\pm \mathrm{SD})$ & $3.9 \pm 1.7$ & $3.8 \pm 1.8$ & 0.842 \\
Diabetes (n, \%) & $27(30.0 \%)$ & $101(22.8 \%)$ & 0.145 \\
Rheumatoid arthritis (n, \%) & $13(14.6 \%)$ & $30(7.0 \%)$ & 0.018 \\
Index revision (n, \%) & $30(33.3 \%)$ & $108(24.4 \%)$ & 0.077 \\
Prior I\&D (n, \%) & $26(28.9 \%)$ & $146(33.0 \%)$ & 0.452 \\
Sinus tract (n, \%) & $25(28.1 \%)$ & $99(22.3 \%)$ & 0.242 \\
Culture at resection arthroplasty (n, \%) & & \\
$\quad$ S. aureus & $33(36.7 \%)$ & $174(39.3 \%)$ & 0.643 \\
CNS* & $26(28.9 \%)$ & $95(21.4 \%)$ & 0.124 \\
$\quad$ Resistant organism & $21(23.3 \%)$ & $66(14.9 \%)$ & 0.048 \\
Streptococcus spp. & $10(11.1 \%)$ & $55(12.4 \%)$ & 0.730 \\
Enterococcus spp. & $8(8.9 \%)$ & $20(4.5 \%)$ & 0.115 \\
Gram-negative organism & $14(15.6 \%)$ & $51(11.5 \%)$ & 0.285 \\
Polymicrobial organism & $17(18.9 \%)$ & $41(9.3 \%)$ & 0.007 \\
Culture negative & $10(11.1 \%)$ & $71(16.0 \%)$ & 0.236 \\
\hline
\end{tabular}

*Coagulase negative staphylococcus 
Table 2 Univariate and multivariate analysis for failure to undergo reimplantation

* Before matching, all confounders in Table 1 were adjusted; after matching, PSM score were

\begin{tabular}{|c|c|c|c|c|c|c|}
\hline & $\begin{array}{l}\text { Reimplantation } \\
(\mathrm{n}, \%)\end{array}$ & P-value & $\begin{array}{l}\text { Non-adjusted } \\
\text { OR }\end{array}$ & $\mathrm{P}$-value & *Adjust OR & P-value \\
\hline \multicolumn{7}{|l|}{ Before matching } \\
\hline $\begin{array}{l}\text { Non-exchange } \\
\text { group }\end{array}$ & $362(81.7 \%)$ & - & Reference & - & Reference & - \\
\hline Exchange group & $63(70.0 \%)$ & 0.012 & $1.92(1.15,3.19)$ & 0.013 & $1.96(1.08,3.53)$ & 0.026 \\
\hline \multicolumn{7}{|l|}{ After matching } \\
\hline $\begin{array}{l}\text { Non-exchange } \\
\text { group }\end{array}$ & $135(76.7 \%)$ & - & Reference & - & Reference & - \\
\hline Exchange group & $61(69.3 \%)$ & 0.196 & $1.46(0.82,2.58)$ & 0.197 & $1.44(0.80,2.60)$ & 0.220 \\
\hline
\end{tabular}


Table 3 Univariate and multivariate analysis for association between spacer exchange and treatment failure

\begin{tabular}{|c|c|c|c|c|c|c|}
\hline & $\begin{array}{l}\text { Failure } \\
(\mathrm{n}, \%)\end{array}$ & P-value & $\begin{array}{l}\text { Non-adjusted } \\
\text { HR }\end{array}$ & P-value & *Adjust HR & P-value \\
\hline \multicolumn{7}{|l|}{ Before matching } \\
\hline $\begin{array}{l}\text { Non-exchange } \\
\text { group }\end{array}$ & $80(22.1 \%)$ & - & Reference & - & Reference & \\
\hline Exchange group & $26(41.3 \%)$ & 0.001 & $2.48(1.42,4.34)$ & 0.002 & $\begin{array}{l}2.05(1 \\
3.89)\end{array}$ & 0.028 \\
\hline \multicolumn{7}{|l|}{ After matching } \\
\hline $\begin{array}{l}\text { Non-exchange } \\
\text { group }\end{array}$ & $31(23.0 \%)$ & - & Reference & - & Reference & - \\
\hline Exchange group & $24(39.3 \%)$ & 0.018 & $2.18(1.13,4.18)$ & 0.019 & $\begin{array}{l}2.23(1.14, \\
4.40)\end{array}$ & 0.020 \\
\hline
\end{tabular}

* Before matching, all confounders in Table 1 were adjusted; after matching, PSM score were adjusted. 


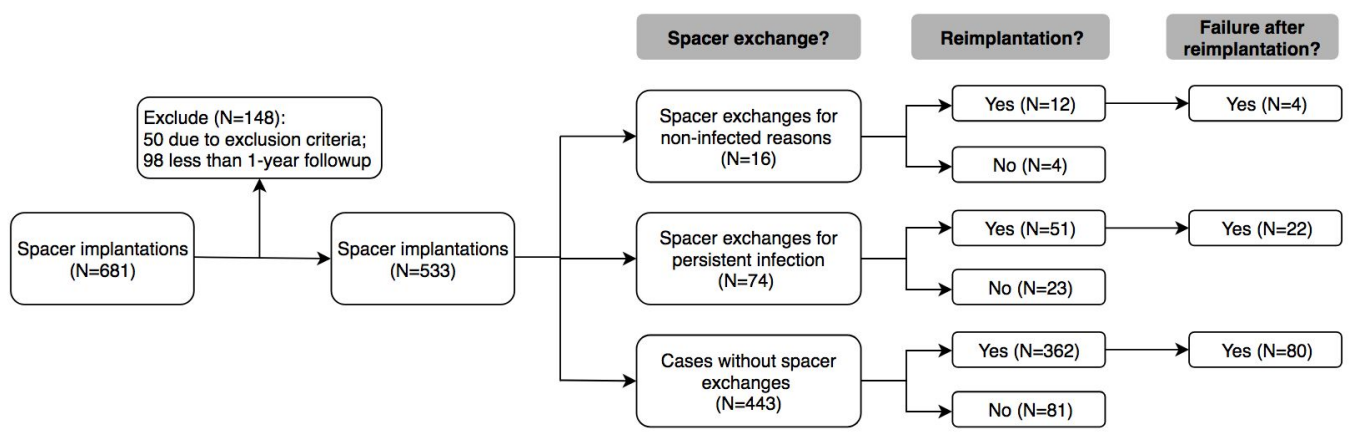



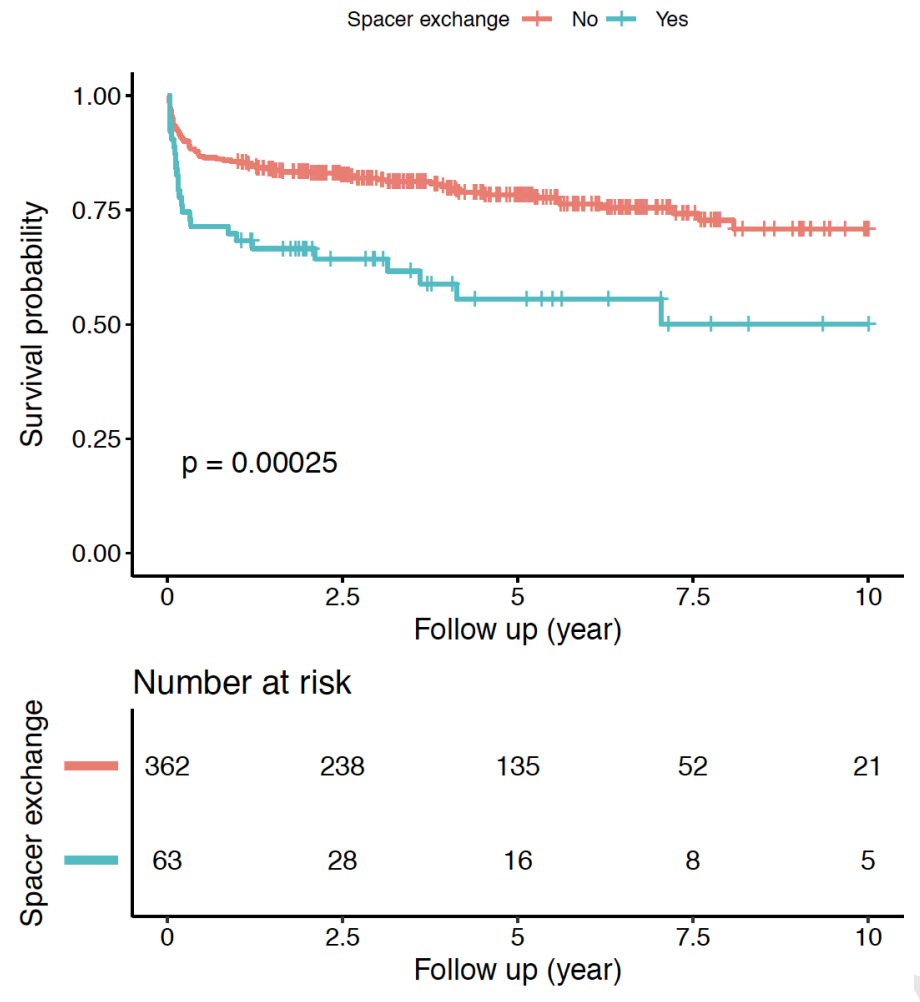

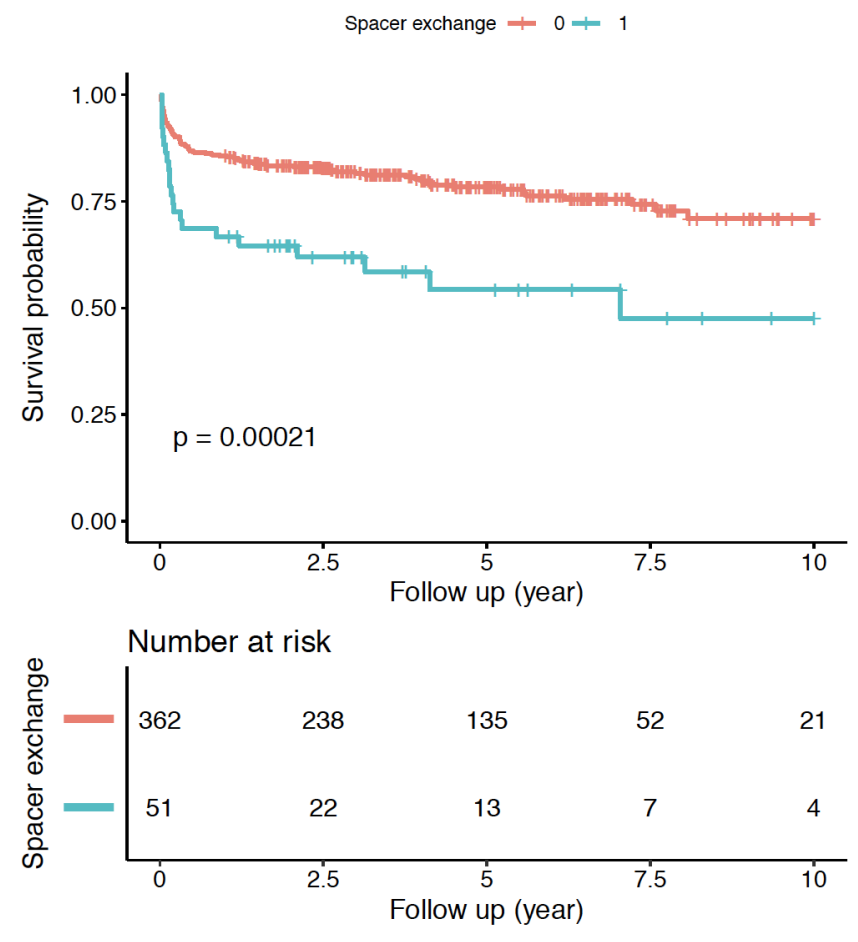

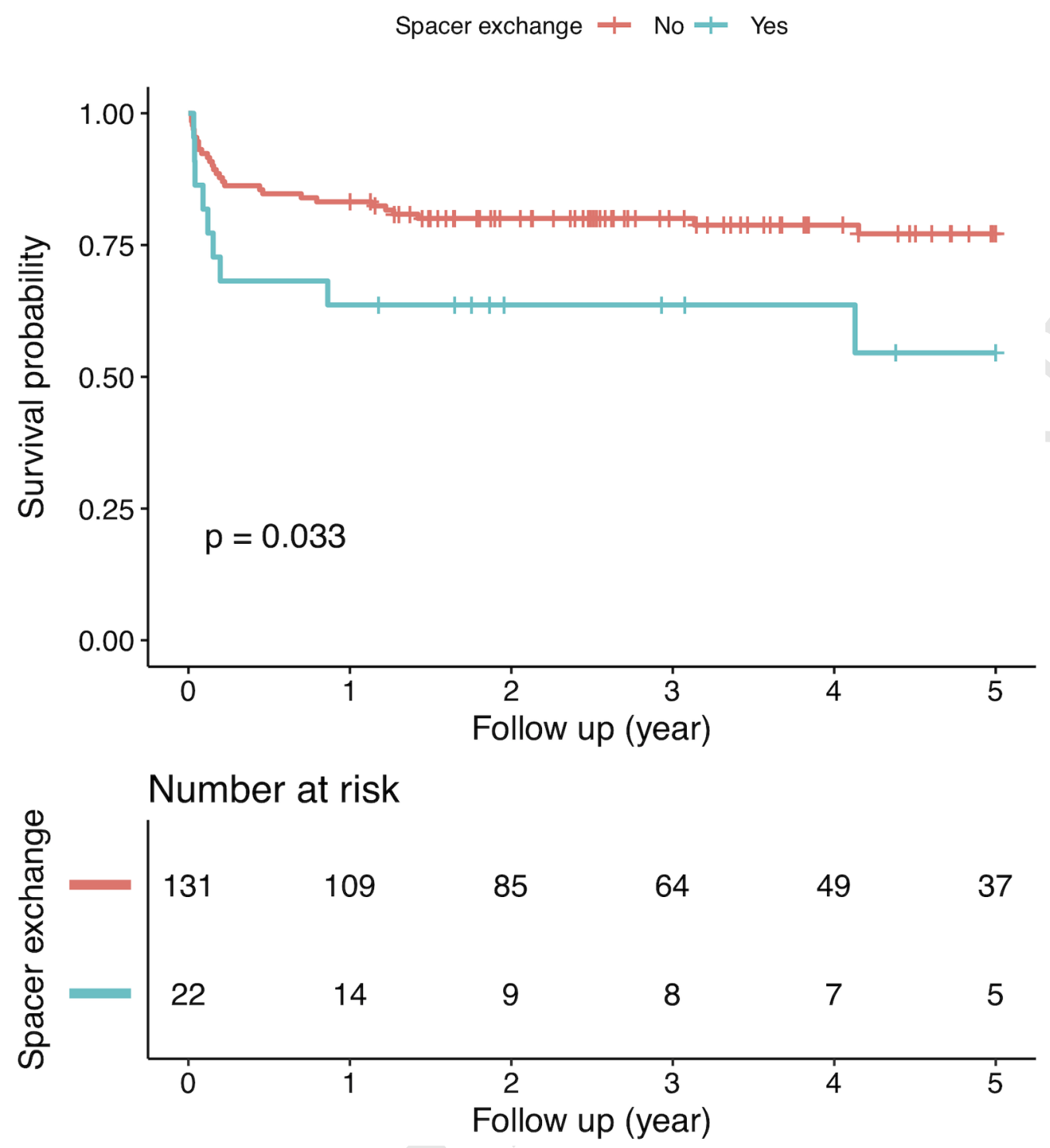


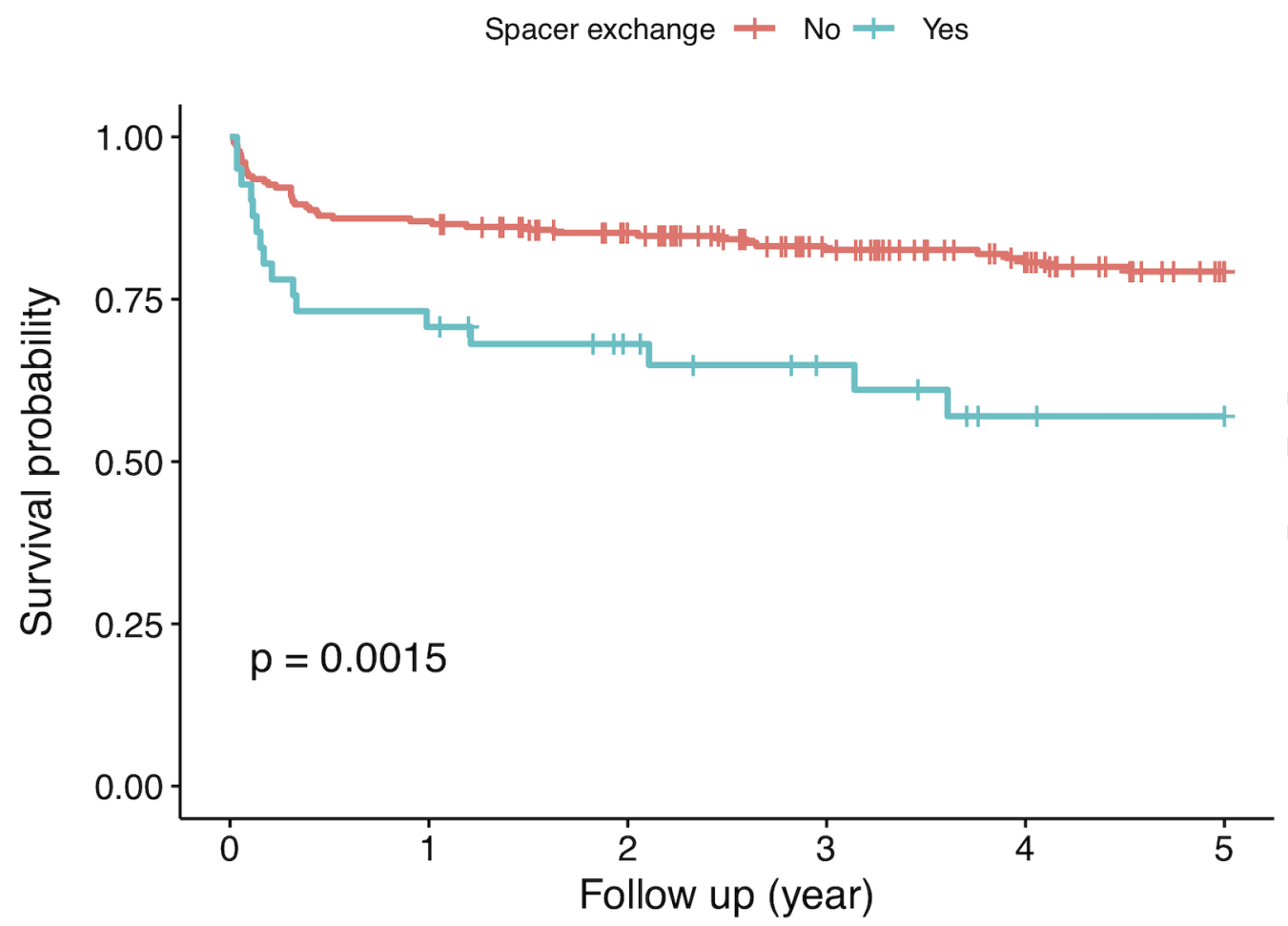

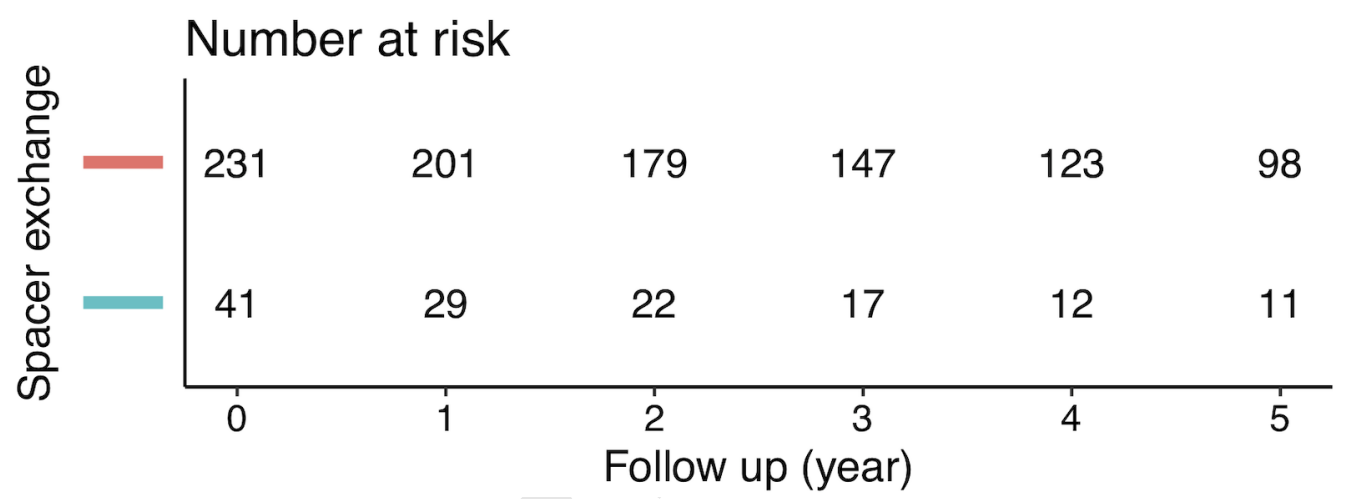




\section{Figure Legend}

Figure 1 Study flowchart

Figure 2 Kaplan-Meier survivorship curve for entire cohort versus controls with treatment failure as an endpoint.

Figure 3 Kaplan-Meier survivorship curve for subgroup of cohort who underwent spacer exchange only for infection (i.e. not for dislocation or other non-infection reasons) versus all controls with treatment failure as an endpoint

Figure 4 Kaplan-Meier survivorship curve for entire cohort versus controls for two-stage exchange arthroplasty after THA PJI with treatment failure as an endpoint

Figure 5 Kaplan-Meier survivorship curve for entire cohort versus controls for two-stage exchange arthroplasty after THA PJI with treatment failure as an endpoint

Appendix Figure 1 Kaplan-Meier implant survivorship curve for entire cohort versus controls after matching

Appendix Figure 2 Kaplan-Meier implant survivorship curve for subgroup of cohort who underwent spacer exchange only for infection (i.e. not for dislocation or other non-infection reasons) versus controls after matching 